\title{
BREAKING CRITERIA FOR LABORATORY EXPERIMENTS BASED ON THE PHASE-TIME-METHOD (PTM)
}

\author{
Kai Irschik ${ }^{1}$, Stefan Schimmels ${ }^{2}$ and Hocine Oumeraci ${ }^{3}$
}

\begin{abstract}
Breaking waves generated by focusing of transient wave packets have been analyzed. By a comparison of video data and gauge measurements the threshold frequency for the use of the PTM as a breaking criterion is derived. The present result is slightly higher than the original value of Zimmermann \& Seymour (2002) for spilling breakers, but confirms the results of Irschik \& Oumeraci (2006). Additionally the use of the zero down-cross period $T_{z}$ instead of the peak period $T_{P}$ is investigated. Both definitions lead for the present conditions with plunging breakers to almost identical results.
\end{abstract}

Keywords: transient wave packet, wave focusing, breaking waves, breaking criterion, Phase-Time-Method

\section{INTRODUCTION}

The breaking of water waves is always connected to high turbulence production rates and therefore significant mixing of water with air and/or sediment. Furthermore, wave breaking can significantly enhance the loads on offshore constructions due to the very high impact when the water hits the structure (e.g. Wienke \& Oumeraci, 2005; Irschik \& Oumeraci, 2006). The exact definition of the breaking point is therefore not only of major interest in oceanography but also an important issue in engineering to develop safe design rules for offshore constructions and coastal protection structures as well as to get a better understanding of the sediment transport processes.

The determination of the incipient wave breaking is, however, not a trivial issue. This is illustrated by the amount of published breaking criteria (e.g. Kamphuis, 1991), which are mostly based on global wave parameters like wave height $H$, wave length $L$ and beach slope $m$. However, these criteria, based on global parameters, are not suitable to detect the breaking of individual waves in an irregular sea state or of a focused wave, like a freak wave for instance.

To overcome this disadvantage, a number of investigations (e.g. Bonmarin, 1989; Duncan et al., 1987; Lader et al., 1998) attempted to set up breaking criteria by using geometric properties of the waves such as the wave front steepness or the vertical asymmetry (ratio of crest height to total wave height). The advantage of these parameters is that they are more specific and can be applied on individual waves e.g. from gauge measurements of the water surface elevation. However, neither the wave front steepness nor the vertical asymmetry provides a general criterion which is valid for all kinds of breaking waves. This could be confirmed by an analysis of measurements in the Large Wave Flume (GWK) of the coastal research center Forschungszentrum Kueste (FZK) where these parameters in the time domain did not apply as a general breaking criterion for all sea states (Irschik \& Oumeraci, 2006). Taking the center of gravity of the area below the wave crest and the wave front as additional parameters also provided no satisfying results. Although the wave front steepness works quite well to predict the breaking point of plunging breakers, it is not possible to define a general criterion based on parameters in the time domain alone, as these are not capable to detect rapid changes of the wave shape. Thus, breaking criteria in the time domain must always be validated for different conditions, e.g. spilling, plunging or collapsing breakers for regular waves, focusing waves, or irregular waves.

A very simple, yet very time consuming method to detect the breaking point of individual waves in a record is the (visual) analysis of video data. Even if this method is rather universal and applies to more or less any type of breaking waves, it implies several disadvantages of which the enormous effort to evaluate the video data image by image represents only one. Furthermore, the need for a reference system to identify the breaking point quantitatively restricts the analysis of video records more or less to well defined situations in the laboratory. But even with a well prepared reference system, the definition of the breaking point, i.e. when the wave really starts breaking, remains rather subjective and might thus lead to fully different results, depending on the skills and experience of the analyst. Therefore, it is obvious that video data can serve as a first start-off or as a good reference for other methods, but a

\footnotetext{
1 Load Department, AREVA Wind GmbH, Am Lunedeich 156, 27572 Bremerhaven, Germany; kai.irschik@AREVA.com

2 Forschungszentrum Kueste (FZK), Merkurstr. 11, 30419 Hannover, Germany, schimmels@fzk-nth.de

3 Leichtweiß-Institute for Hydraulic Engineering, Beethovenstr. 51a, 38106 Braunschweig, Germany, h.Oumeraci@tu-bs.de
} 
reliable and unique definition of the breaking point requires the time series analysis of water surface elevation recorded from gauge measurements.

Huang et al. (1992) were the first to show the potential of the Hilbert transform in highlighting local properties of steep, non-linear water waves. The Hilbert transform of a time series allows deriving a time dependent frequency of the original signal which describes the variation of the local frequency from the mean frequency of the record. Huang et al. (1992) recognized a jump of this Hilbert frequency on the wave front, just below the wave crest when the wave gets very steep or starts to break. Based on the findings of Huang et al. (1992), Zimmermann \& Seymour (2002) developed the Phase-TimeMethod (PTM) to detect deep water spilling breakers in a single point wave record. They found that when the Hilbert frequency exceeds a certain threshold, the corresponding wave is breaking or will irrevocably break, respectively. The chosen threshold of about $3 \mathrm{rad} / \mathrm{s}\left(f_{\text {thres }}\left(T_{P}\right) \approx 0.48 \mathrm{~Hz}\right)$ corresponds to about $85 \%$ of the peak frequency of the underlying spectrum and was assumed to vary with the peak frequency. However, although the peak frequencies of the investigated spectra of Zimmermann \& Seymour (2002) ranged between $0.48 \mathrm{~Hz}$ and $0.63 \mathrm{~Hz}$, with the general threshold of about $0.48 \mathrm{~Hz}$ more than $95 \%$ of all breaking waves could be detected.

Irschik \& Oumeraci (2006) also applied the PTM in a slightly modified way to analyze the effect of breaker types on breaking wave loads on a pile. In this study transient wave packets on a horizontal bottom as well as regular and irregular breaking waves on a 1:10 sloping bottom were generated and a threshold frequency of $0.7 \mathrm{~Hz}$ instead of $0.48 \mathrm{~Hz}$ suggested by Zimmermann \& Seymour (2002) was found to work best. The motivation for the present analysis is this quite remarkable discrepancy as well as the fact that the work of Irschik \& Oumeraci (2006) was focused on the wave loads rather than the PTM itself. The main goal of this paper is an accurate determination of the threshold frequency by a thorough analysis of breaking focused wave packets in a specifically designed laboratory experiment.

After a short revisit of the Phase-Time-Method in the next section the model setup and the methodology of the present investigation are explained. By a comparison of video data and gauge measurements an upper and lower bound for the threshold frequency are defined and a general value will be derived which is assumed to be valid at least for the present type of intermediate and shallow water breaking waves. Finally the PTM with the found threshold value will be applied to a practical example to show its advantages compared to a visual load case analysis.

\section{PHASE-TIME-METHOD (PTM)}

The Phase-Time-Method (PTM) has first been presented by Zimmermann \& Seymour (2002) and is based on a time dependent frequency of the water surface elevation derived from the Hilbert transform of the original time signal. Before the estimation of the breaking point is further illustrated the basic idea of the Hilbert frequency $f_{H T}$ and the way to obtain it from a time series of the water surface elevation shall shortly be repeated.

Any time series can be written in the following form:

$$
\eta(t)=\sum_{n=0}^{\infty} a_{n} \cos (n \sigma t)+b_{n} \sin (n \sigma t)
$$

so that the corresponding Hilbert transform is

$$
h(t)=\sum_{n=0}^{\infty} a_{n} \sin (n \sigma t)-b_{n} \cos (n \sigma t) .
$$

From the original time signal and its Hilbert transform an analytical signal can be constructed:

$$
\psi(t)=\eta(t)+i h(t)
$$

where the time dependent phase function is given by

$$
\phi(t)=\arctan \left(\frac{h(t)}{\eta(t)}\right) .
$$

The Hilbert frequency is finally obtained as the time derivative of the phase function

$$
f_{H T}(t)=\frac{\partial \phi}{\partial t},
$$


and basically describes the variation of the instantaneous frequency from the mean frequency of the signal and is therefore a measure for nonlinearity in a signal. For a pure sinusoidal wave, i.e. a linear signal, the Hilbert frequency is in fact a constant and represents the (mean) frequency of the wave. More details on the Hilbert transform and the Hilbert frequency can be found in the original work of Huang et al. (1992) or Zimmermann \& Seymour (2002).

The breaking of water waves is always connected to a high nonlinearity which increases while the wave shoals and eventually breaks. The Hilbert frequency is therefore excellently suited to detect breaking waves in a time signal when a certain threshold is exceeded, i.e. nonlinearity becomes so high that the wave breaks. If the breaking process takes place within an array of wave gauges it is possible to determine the breaking point from the individual time series, as done for the present investigation. The basic procedure is sketched in Figure 1.

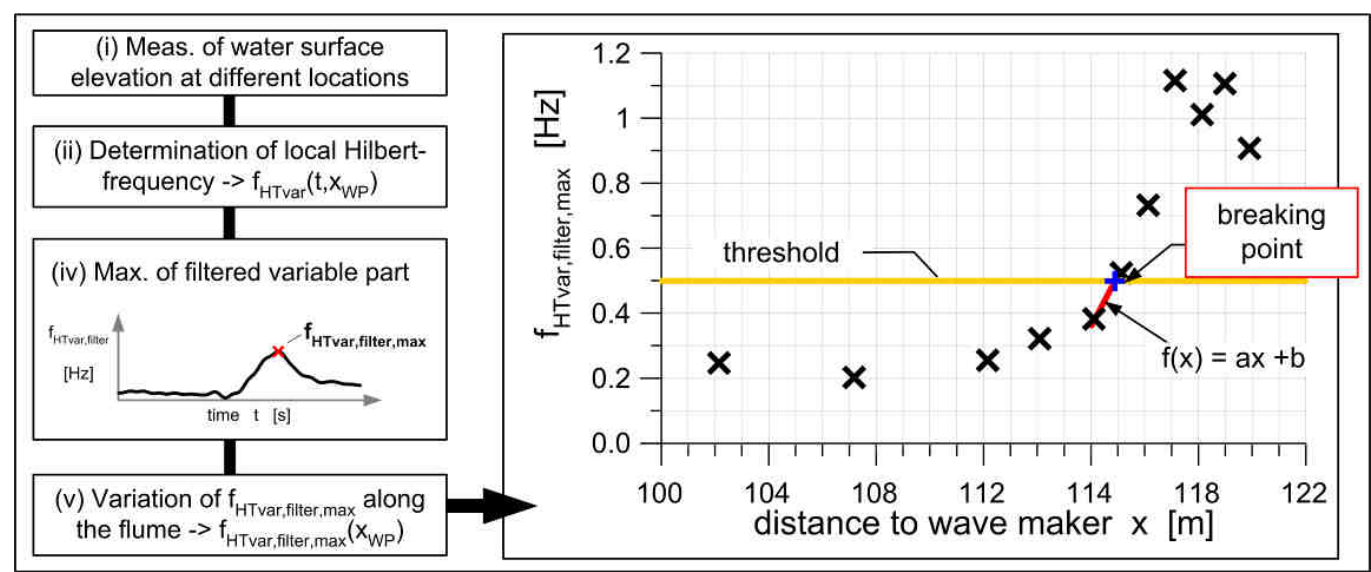

Figure 1. Estimation of the incipient breaking by using the PTM and the maxima of the Hilbert-frequencies for a fictitious plunging breaker. The variable local Hilbert-frequency highlights the evolution of the breaking process and can be used as a breaking criterion in combination with an experimental threshold.

The individual steps can be summarized as follows:

i. Measurement of the water surface elevation at different locations $x$ along the flume. The raw data is low-pass filtered with $45 \mathrm{~Hz}$ to eliminate the noise from the electrical grid to get:

$$
\eta(t, x) \quad[\mathrm{m}]
$$

ii. Calculation of the local Hilbert-frequency $f_{H T}(t, x)$ and the variable frequency $f_{H T \text { var }}(t, x)$ by considering the zero-down cross period $T_{z}=1 / f_{T_{z}}$ for every single wave:

$$
f_{H T v a r}(t, x)=f_{H T}(t, x)-f_{T z}(x) \quad[\mathrm{Hz}]
$$

or by considering the peak period $T_{P}=1 / f_{T_{p}}$ of the underlying spectrum:

$$
f_{H T v a r}(t, x)=f_{H T}(t, x)-f_{T p}(x) \quad[\mathrm{Hz}]
$$

iii. Filtering of $f_{H T \text { var }}(t, x)$ with a moving average filter in order to remove the remaining spurious oscillations:

$$
\begin{aligned}
& f_{\text {HTvar, filter }}(t, x)=\frac{1}{2 m+1} \sum_{i=-m}^{N=m+1} f_{\text {HTvar }}\left(t_{i}, x\right) \quad[\mathrm{Hz}] \\
& \text { with: } \quad m=\frac{T_{\text {front }}}{20 \cdot \Delta t}
\end{aligned}
$$

iv. Determination of the maximum instantaneous Hilbert frequency $f_{H T \text { var, filter }}$ :

$$
f_{H T \text { var, filter, } \max }(x)=\max \left[f_{H T \text { var, filter }}(t, x)\right] \quad[H z]
$$


v. Check if the threshold frequency is exceeded:

$$
f_{H T \text { var, }, \text { filter, } \max }(x) \stackrel{?}{>} \mathrm{f}_{\text {thres }}
$$

vi. Estimation of the breaking point $x_{b}$

$$
\begin{aligned}
& \left(f_{H T \text { var, filter, } \text { max }}\left(x_{i-1}\right)+\frac{f_{H T \text { var, }, \text { filter, } \text { max }}\left(x_{i}\right)-f_{H T \text { var, }, \text { ilter, } \text { max }}\left(x_{i-1}\right)}{x_{i}-x_{i-1}} \cdot x_{b}\right)-\mathrm{f}_{\text {thres }} \stackrel{!}{=} 0 \\
& \text { where: } \quad f_{H T \text { var, filter, } \text { max }}\left(x_{i-1}\right)<\mathrm{f}_{\text {thres }} \wedge f_{H T \text { var, filter, } \text { max }}\left(x_{i}\right)>\mathrm{f}_{\text {thres }}
\end{aligned}
$$

Zimmermann \& Seymour (2002) point out several prerequisites for the use of the PTM. These are (i) the sampling rate of the time signal, (ii) a limit of the water surface elevation to eliminate mathematical artifacts in the region of the still water level and (iii) the validated threshold, i.e. the breaking criterion itself.

The required sampling rate for the PTM to provide reasonable results is actually the reason why the method might be limited to laboratory experiments. Zimmermann \& Seymour (2002) point out that the minimum sampling rate should be 25 times the peak frequency and a sampling rate of 50 times the peak frequency would greatly improve the results. Thus, if in-situ measurements are available with sampling rates of approximately $5-10 \mathrm{~Hz}$, the time series could be analyzed with the PTM in the same manner as presented here. To be on the safe side the data for the present work was sampled with $100 \mathrm{~Hz}$ and had therefore to be low pass filtered in order to remove the noise from the electrical grid. As mentioned above the filter frequency was set to $f_{\text {cut low-pass }}=45 \mathrm{~Hz}$.

Due to its definition the Hilbert frequency becomes unbound if the water surface elevation $\eta$ approaches zero and its Hilbert transform $h$ is small as well. However, this mathematical artifact has been observed in connection with multiple breaking transient wave packets or irregular sea states as investigated by Zimmermann \& Seymour (2002). Here transient wave packets on a horizontal bottom are analyzed and the phenomena can only be observed well before and after the focusing event when the water surface is rather calm. Nevertheless, to avoid any outliers in the present data and to make the methodology as universal as possible, the data has been additionally filtered with a moving average filter covering $1 / 20^{\text {th }}$ of the wave front period: $T_{\text {filter }}=T_{\text {front }} / 20[\mathrm{~s}]$.

The third prerequisite - the threshold frequency - is the major goal of this work and will be analyzed in the next sections.

\section{TEST SET-UP IN THE LARGE WAVE FLUME (GWK)}

The experimental tests were performed in the Large Wave Flume (GWK) of the coastal research center Forschungszentrum Kueste (FZK) in Hannover, Germany. The GWK is $300 \mathrm{~m}$ long, $5 \mathrm{~m}$ wide and $7 \mathrm{~m}$ deep, the water depth for the present experiments was always $4.0 \mathrm{~m}$ and the analyzed nonbreaking and breaking waves had a peak period of the underlying spectrum of $T_{p}=6.0 \mathrm{~s}$ and varying heights and focus points between about $100 \mathrm{~m}$ and $112 \mathrm{~m}$.

The main goal is to get a sound definition of the threshold frequency for the PTM by exemplarily using focused wave packets was basically achieved by comparing visually observed breaking points with those determined by the PTM as described above. Therefore, two video cameras were installed on each side of the flume with a drawn grid on one sidewall and the wave gauges on the opposite wall serving as a reference for the visual estimation of the breaking point. To determine the breaking point from direct gauge measurements of the water surface elevation with the PTM a very dense array of wave gauges was installed with varying distances from $5.0 \mathrm{~m}$ to $0.5 \mathrm{~m}$. The exact positions of the wave gauges were at $x=100,105,106,107,108,109,109.75,110.25,110.75,111.25,114$ and $120 \mathrm{~m}$ from the wave maker. Wave gauge number 1 in Figure 2 is located at $x=105 \mathrm{~m}$.

The model set-up is sketched in Figure 2. The top view of the flume in the upper part of the figure is scaled by about 1:150, so only the central gauges between 105 and $111.25 \mathrm{~m}$ are displayed. The monopile in the middle of the flume has a diameter of $50 \mathrm{~cm}$ and is placed at $x=111.00 \mathrm{~m}$. The graphs at the lower left show some sample data of the water surface elevation at different gauges which served as input for the PTM and the images in the lower right are snapshots from the two video cameras in order to illustrate on what data the visual estimation of the breaking point was based. 

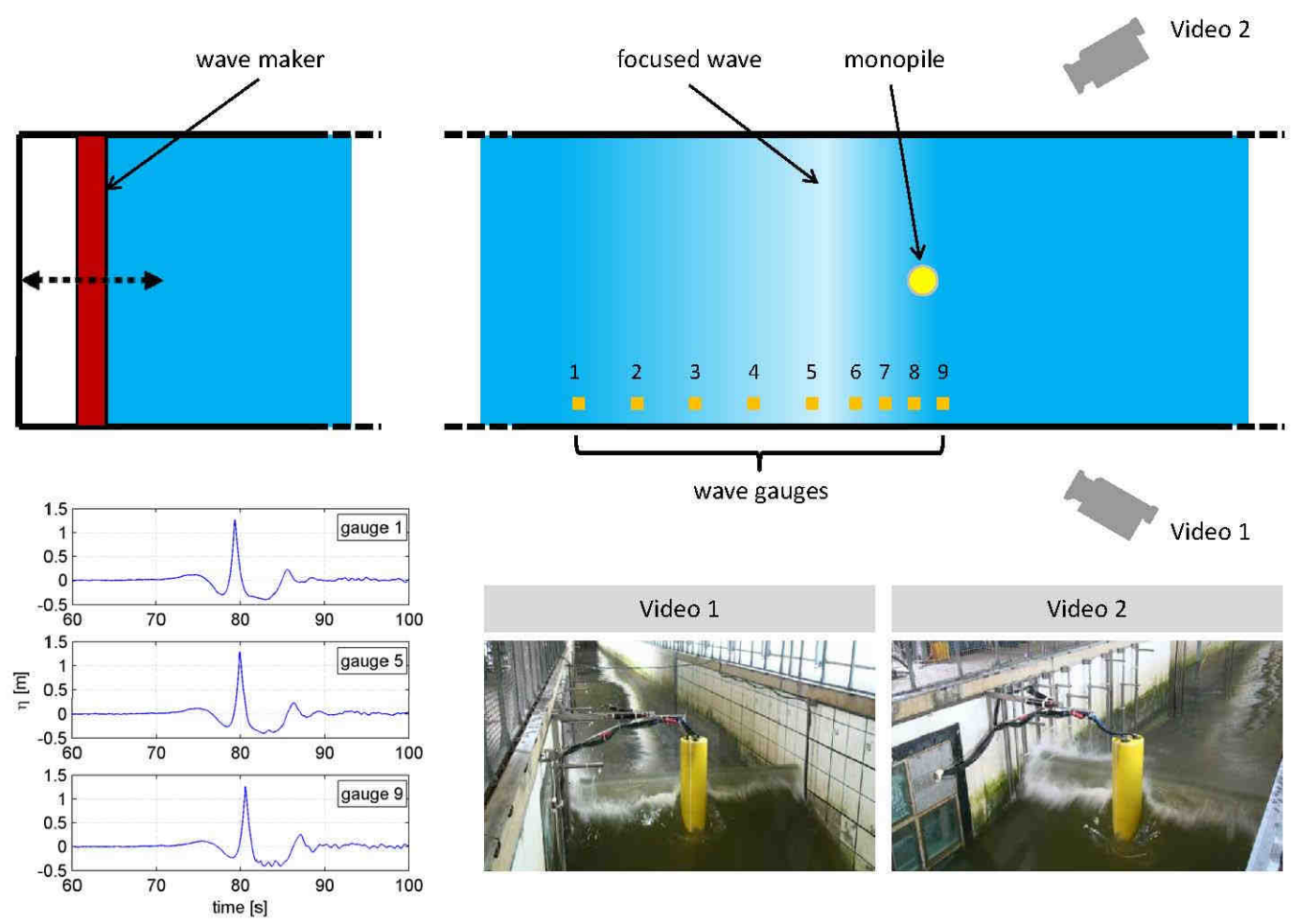

Figure 2. Test set-up and example wave records in the Large Wave Flume (GWK) in Hannover, Germany.

The parameters of the investigated waves for this study are summarized in Table 1. As mentioned above the wave height and the concentration point were varied in order to generate different breaking wave conditions. The wave with the smallest height of $1.50 \mathrm{~m}$ is very steep but did not break. It could therefore serve as the lower limiting case for the determination of the frequency threshold, as will be explained in the next section.

Table 1. Wave parameters tested in GWK and location of the visually estimated breaking point

\begin{tabular}{|c|c|c|c|c|c|}
\hline $\begin{array}{l}\text { test no. } \\
\text { [-] }\end{array}$ & $\begin{array}{c}\text { wave type } \\
{[-]}\end{array}$ & $\begin{array}{c}\text { wave } \\
\text { height } \\
\text { H } \\
{[\mathrm{m}]}\end{array}$ & $\begin{array}{c}\text { peak } \\
\text { period } \\
T_{p} \\
{[s]} \\
\end{array}$ & $\begin{array}{l}\text { water } \\
\text { depth } \\
\text { d } \\
{[\mathrm{m}]}\end{array}$ & $\begin{array}{c}\text { visually estimated } \\
\text { breaking point } \\
\mathrm{x}_{\mathrm{b}} \\
{[\mathrm{m}]}\end{array}$ \\
\hline 2010052805 & non-breaking & 1.50 & 6.00 & 4.00 & - \\
\hline 2010053106 & Breaking & 1.60 & 6.00 & 4.00 & 111.7 \\
\hline 2010052807 & Breaking & 1.60 & 6.00 & 4.00 & 109.6 \\
\hline 2010052808 & Breaking & 1.60 & 6.00 & 4.00 & 108.2 \\
\hline 2010052809 & Breaking & 1.90 & 6.00 & 4.00 & 101.2 \\
\hline 2010052810 & Breaking & 1.90 & 6.00 & 4.00 & 103.3 \\
\hline 2010052811 & Breaking & 1.80 & 6.00 & 4.00 & 105.2 \\
\hline 2010052812 & Breaking & 1.80 & 6.00 & 4.00 & 106.8 \\
\hline 2010053101 & Breaking & 1.70 & 6.00 & 4.00 & 109.9 \\
\hline 2010053102 & Breaking & 1.70 & 6.00 & 4.00 & 110.8 \\
\hline 2010053104 & Breaking & 1.80 & 6.00 & 4.00 & 105.3 \\
\hline 2010053105 & Breaking & 1.80 & 6.00 & 4.00 & 107.8 \\
\hline 2010053106 & Breaking & 1.80 & 6.00 & 4.00 & 109.3 \\
\hline
\end{tabular}


All other waves were breaking with different characteristics depending on the wave height and the concentration point. The visually estimated breaking points result from an analysis of the video data from both cameras. Three different people have made an independent estimation of the breaking point with some expected deviation of about $1.5 \mathrm{~m}$ at most. Even if this discrepancy is not very large it clearly shows the drawback of such kind of analysis, which is always subjective by nature. The values in the last column of Table 1 represent the distance of the breaking point from the wave paddle and are obtained from the average values of all three independent estimations. They will be used as a reference for the determination of the threshold frequency as shown in the next section.

In the visual analysis of the breaking point it has been noted, that the breaker tongue does not occur simultaneously over the whole crest of the wave. Thus, one has to consider the location of the measurement of the water surface elevation as illustrated in Figure 3. Even if the video shows the whole wave, only the sketched reference plane of the wave gauges is of interest in the analysis of the incident breaking.

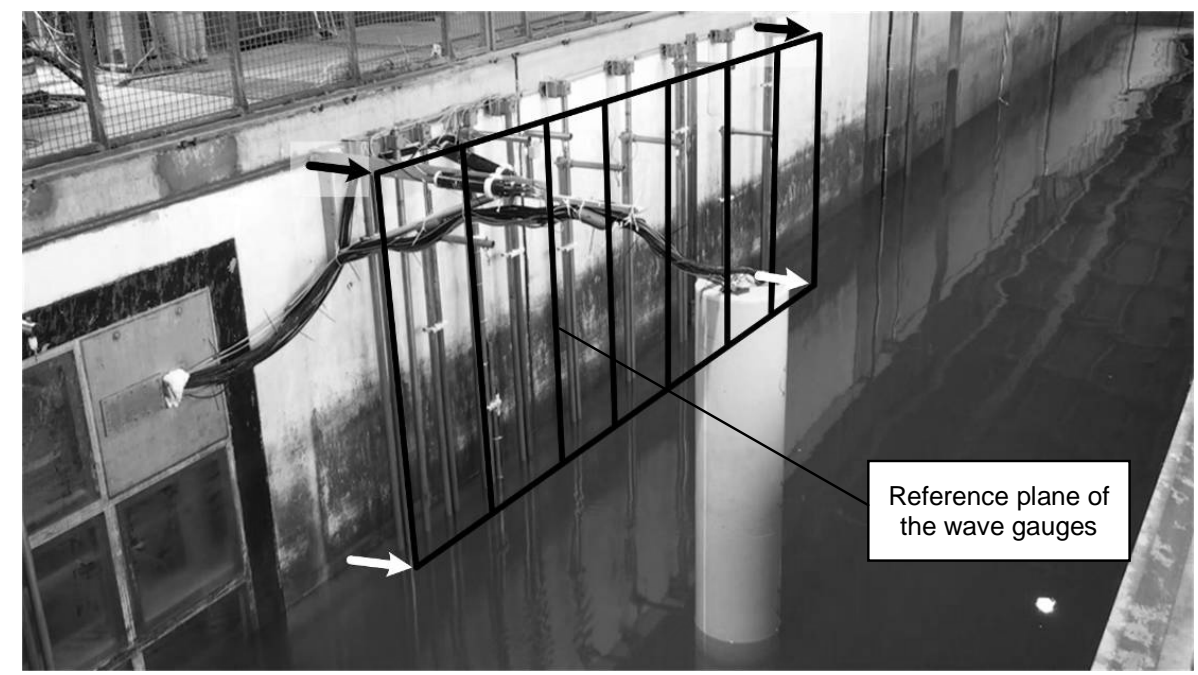

Figure 3. Reference plane of the wave gauge measurements, which is ca. $50 \mathrm{~cm}$ off the wall. 


\section{THRESHOLD DETERMINATION}

The goal of the threshold determination is to find a threshold that is high enough to avoid identifying a non-breaking wave as a breaking wave, but that is low enough to detect all breaking waves. Therefore, first non-breaking waves are analyzed to estimate a lower bound of the threshold frequency. Then the upper bound is defined by all breaking waves.

\section{Non-breaking steep waves - threshold lower bound}

The wave in Figure 4 was classified as a non-breaking wave. The snapshots of the video records show the transient wave packet at different positions in the flume. The measured water surface elevation and the modified Hilbert-frequencies are given as well. While the pictures show the surface variation in space, the wave gauges measure the variation in time at a given location. To show the variation of the results when using eq. (7) or eq. (8) the variation of the local frequency for both equations is plotted.

The first wave in Figure 4 increases in steepness when traveling through the measurement section and the last picture at $x=111.25 \mathrm{~m}$ indicates that breaking will take place immediately after moving forward along the flume. The second wave reaches its highest steepness at the beginning of the sequence. In the first snapshoot it seems that the wave will break, but then it starts to decompose without breaking. Thus, these examples provide a lower bound of the threshold for the breaking criterion; this means the threshold must be higher than the frequencies plotted and must not identify these waves as breaking waves.

$$
\begin{gathered}
f_{\text {thres }}\left(T_{P}\right) \stackrel{!}{>} 0.52 \quad[\mathrm{~Hz}] \\
f_{\text {thres }}\left(T_{z}\right) \stackrel{!}{>} 0.41 \quad[\mathrm{~Hz}]
\end{gathered}
$$

Using $T_{z}$ instead of $T_{p}$ shows the deviation of the local frequency from the global wave period.

\section{Breaking waves - threshold upper bound}

Examples of breaking waves are provided in Figure 5 and Figure 6. In both cases the waves shoal and a breaker tongue develops at the wave crest. Simultaneously, the time history of the Hilbertfrequency develops a peak, which gets higher the closer the wave reaches the breaking point.

For the determination of a threshold frequency, only the maxima up to the visually determined breaking points are considered. The lowest of these values is:

$$
\begin{aligned}
& f_{\text {thres }}\left(T_{P}\right) \stackrel{!}{<} 0.71 \quad[\mathrm{~Hz}] \\
& f_{\text {thres }}\left(T_{z}\right) \stackrel{!}{<} 0.62 \quad[\mathrm{~Hz}]
\end{aligned}
$$

This means that if the threshold frequency is set to $f_{\text {thres }}\left(T_{p}\right)=0.7$ or $f_{\text {thres }}\left(T_{z}\right)=0.6$, all analyzed waves would have been correctly identified as breaking waves. 


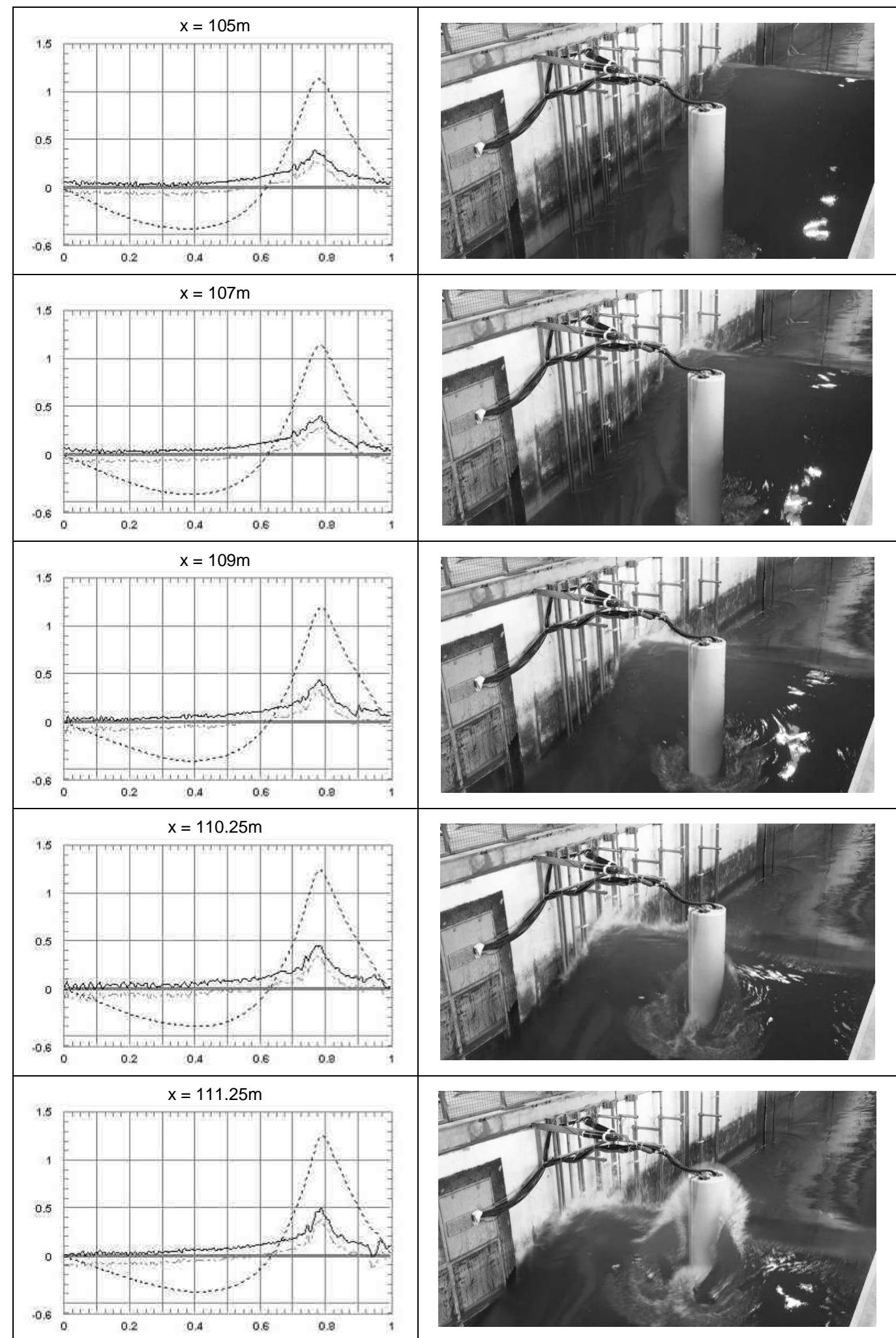

Figure 4. Variation of the wave form in time and space. Left column: Time series of the water surface

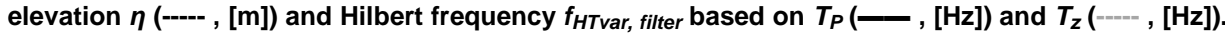
Right column: Snapshots of the wave when the wave crest was at the corresponding gauge positions. Test 2010052805. 


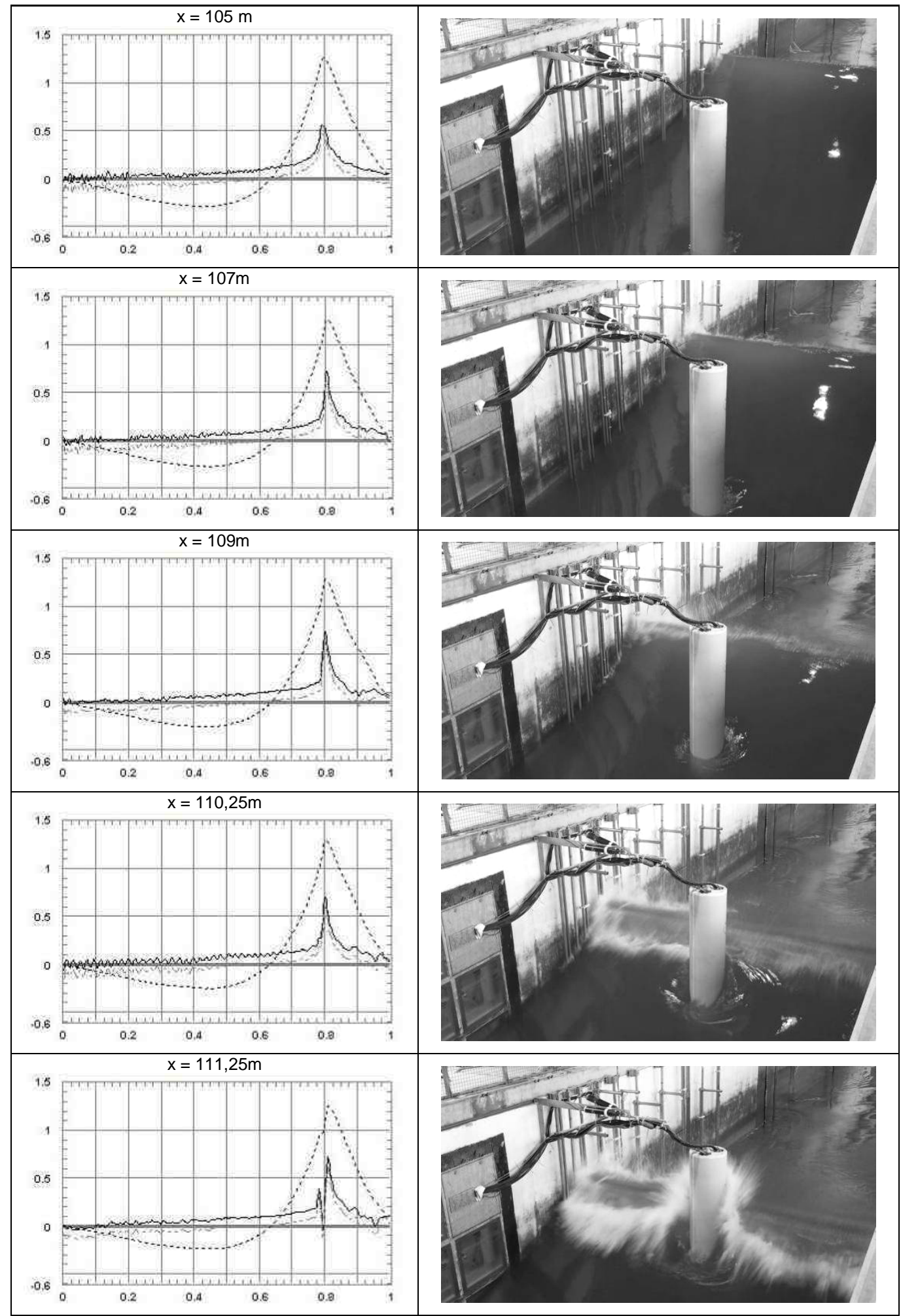

Figure 5. Variation of the wave form in time and space. Left column: Time series of the water surface

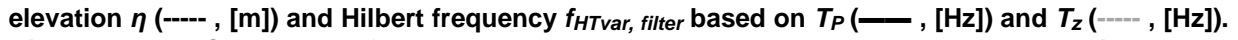
Right column: Snapshots of the wave when the wave crest was at the corresponding gauge positions. Test 2010052808. 


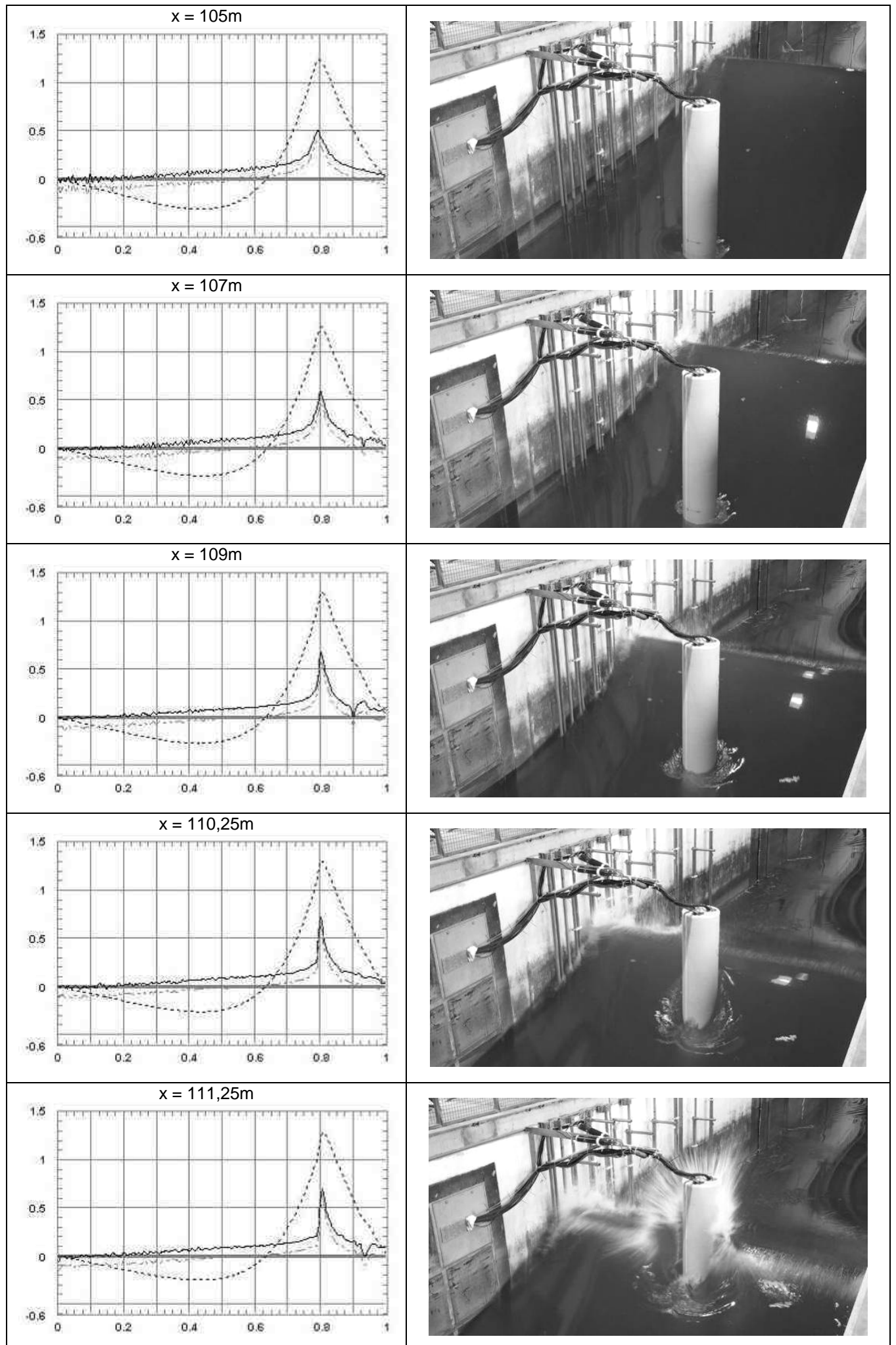

Figure 6. Variation of the wave form in time and space. Left column: Time series of the water surface elevation $\eta(---$, , $[\mathrm{m}])$ and Hilbert frequency $f_{H T v a r}$, filter based on $T_{P}(-,[\mathrm{Hz}])$ and $T_{z}(----,[\mathrm{Hz}])$. Right column: Snapshots of the wave when the wave crest was at the corresponding gauge positions. Test 2010053101. 


\section{Sensitivity of PTM results against variation of the threshold and comparison with visual analysis}

In the previous sections the upper and lower bound of the threshold have been determined. Here the sensitivity of the results on the variation of the threshold frequency within the boundaries is shown in Figure 7. The visual analysis has been used as a reference. Thus, the relative breaking point of $x=0 \mathrm{~m}$ would be identical to the reference. A negative value means that the PTM result is shifted to the wave paddle. Only waves that break within the flume region between $105 \mathrm{~m}$ and $111 \mathrm{~m}$ - where the gauges are very close together - are considered to guarantee a high resolution in space of the signal. The maximum distance between successive wave gauges is $1 \mathrm{~m}$ (see Figure 2).

The visual analysis has been performed by three different persons. The mean value of the results is defined as the breaking point. It is obvious from Figure 7 that the definition of the breaking point varies for the individual persons. Surprisingly, there is no clear trend for any person visible.

Together with the results of the visual analysis, the breaking point of the PTM for different threshold frequencies based on $T_{z}$ is plotted in Figure 7. As expected, the breaking point is closer to the wave paddle for the lowest threshold and farther away for higher thresholds. Nevertheless, the evolution of the waves occurs differently, i.e. the distance between the estimated breaking points changes from wave to wave. For example wave 3 shows equally spaced distances between the breaking points for the various threshold frequencies. This wave shoals smoothly along the flume. In contrast, for wave 1 rapid changes are detected and the wave shape changes for certain steps.

The first important result from Figure 7 is that all breaking points estimated with the PTM, independent of the threshold frequency, are in front of the visually determined breaking point, i.e. closer to the wave paddle. This would not be a disadvantage of the method as long as the offset to the reference is constant. But then the variation of the relative breaking point highly depends on the threshold frequency. The standard deviation of the offset is highest for the lowest threshold. It decreases up to a threshold frequency of $f_{\text {thres }}\left(T_{Z}\right)=0.60 \mathrm{~Hz}$. The frequency $f_{\text {thres }}\left(T_{Z}\right)=0.62 \mathrm{~Hz}$ shows one outlier. Thus, the threshold should be defined to:

$$
f_{\text {thres }}\left(T_{z}\right)=0.60[\mathrm{~Hz}]
$$

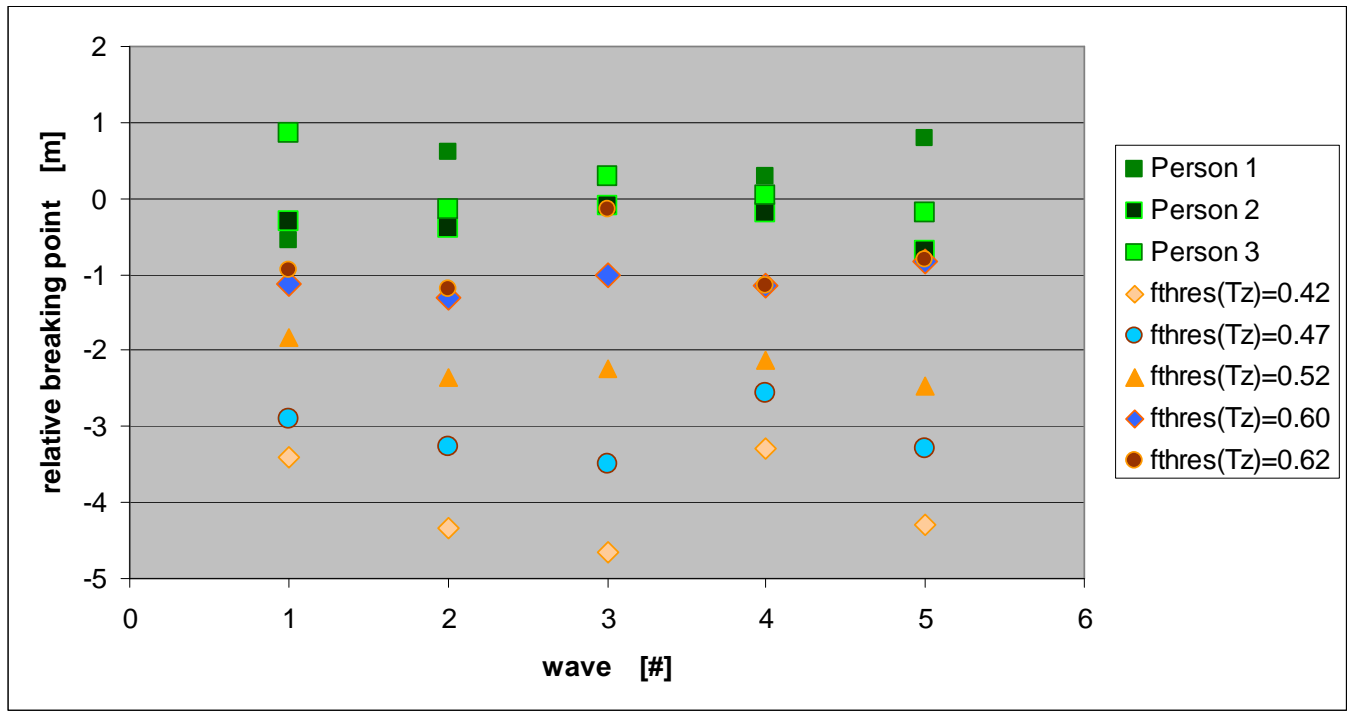

Figure 7. Comparison of the PTM results for different threshold frequencies $f_{\text {thres }}\left(T_{z}\right)$ with visually estimated breaking points.

Figure 8 shows the same analysis for different threshold frequencies based on $T_{P}$. The distribution of the breaking points coincides in general with Figure 7, whereas the offset from the reference to the PTM results is slightly higher. Following the argument of a constant offset, the threshold based on $T_{P}$ should be:

$$
f_{\text {thres }}\left(T_{P}\right)=0.60[\mathrm{~Hz}]
$$


This is somewhat surprising, as this value represents the mean of the previously estimated upper and lower bound. Whereas for the value based on $T_{z}$ is just slightly lower than the upper bound.

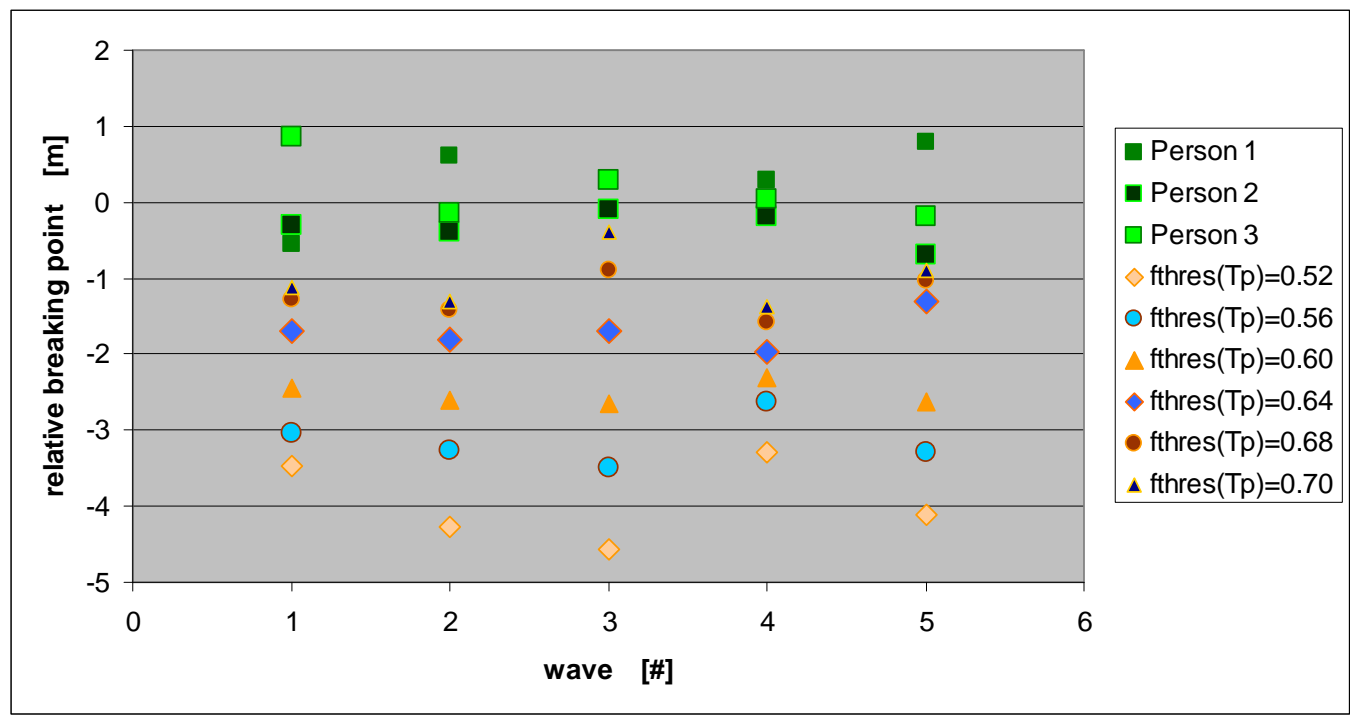

Figure 8. Comparison of the PTM results for different threshold frequencies $f_{\text {thres }}\left(T_{p}\right)$ with visually estimated breaking points.

\section{APPLICATION OF BREAKING CRITERION TO LOAD CASE SEPARATION}

In this section the measured total wave force on a slender cylinder will be used as an application of the PTM. The measurements have been taken from Wienke \& Oumeraci (2005) for five loading cases (LC1-LC5). The normalized total wave force is plotted against the visually estimated load case in Figure 9. According to the definition of Wienke \& Oumeraci (2005) the waves are breaking far in front of the cylinder for LC 1 \& LC2, just in front of the cylinder front for LC 3, right at the cylinder for LC4 and behind the cylinder for LC 5.

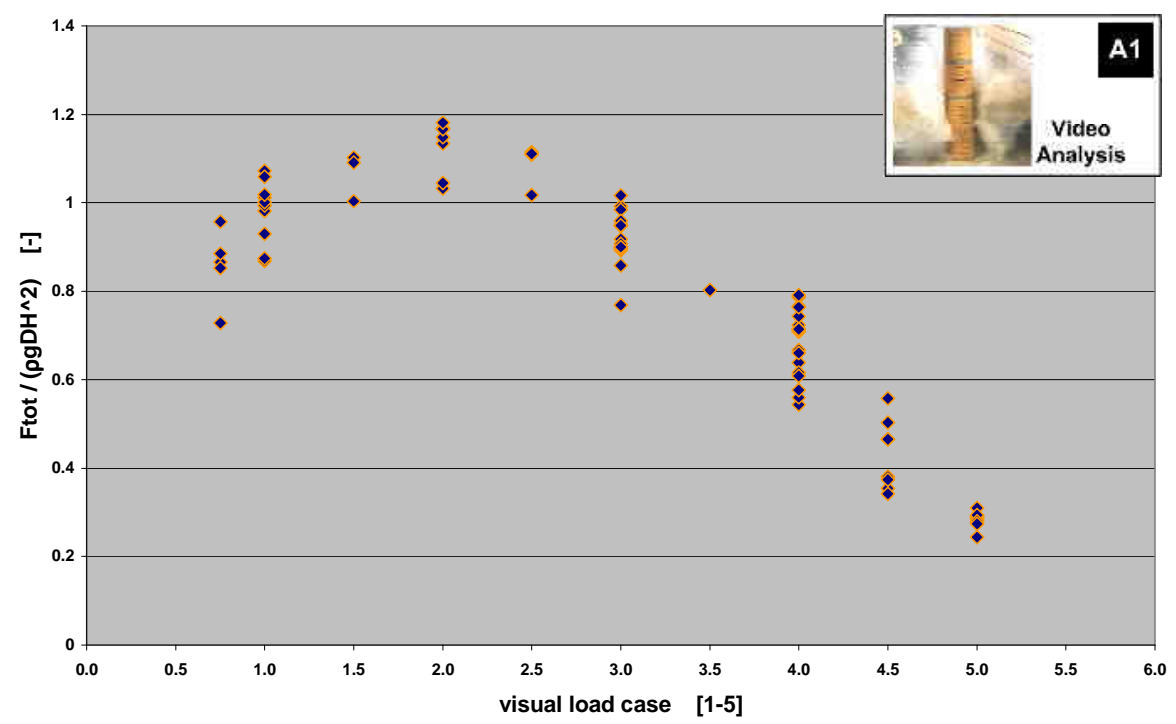

Figure 9. Non-dimensional measured total wave force against visually estimated load cases (Wienke \& Oumeraci, 2005) : LC $\leq 2.5$ : wave breaks far in front of cylinder; LC = 3: Wave breaks just in front of cylinder front; $L C=4$ : wave breaks at the cylinder; $L C=5$ : Non-breaking steep wave, i.e. wave breaks behind the cylinder. 


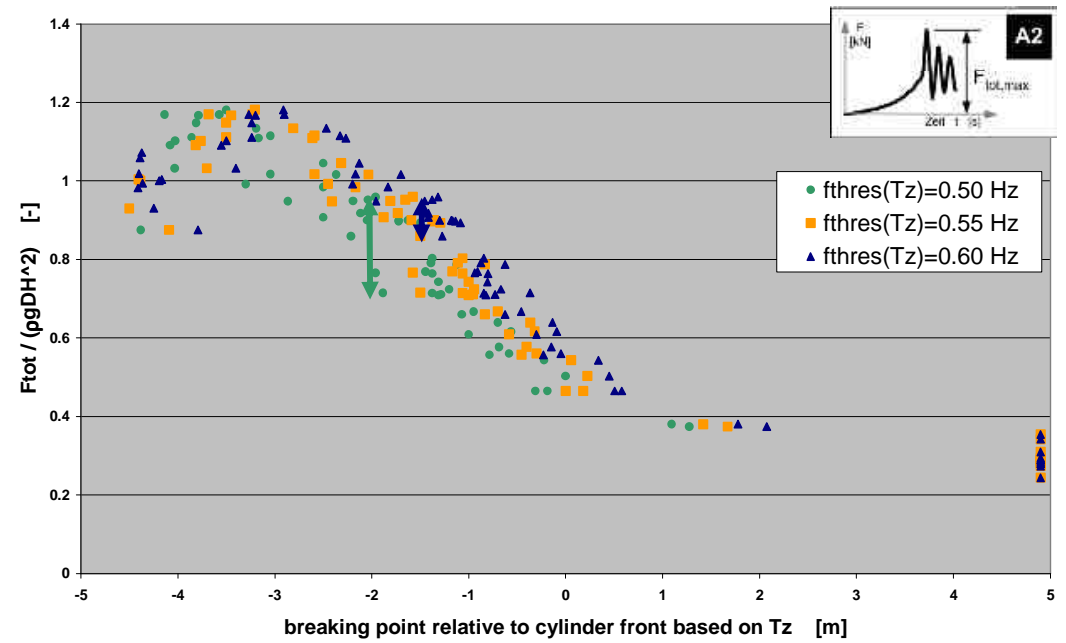

Figure 10. Non-dimensional measured total wave force against relative breaking point. Here PTM based on $T_{z}$ has been used.

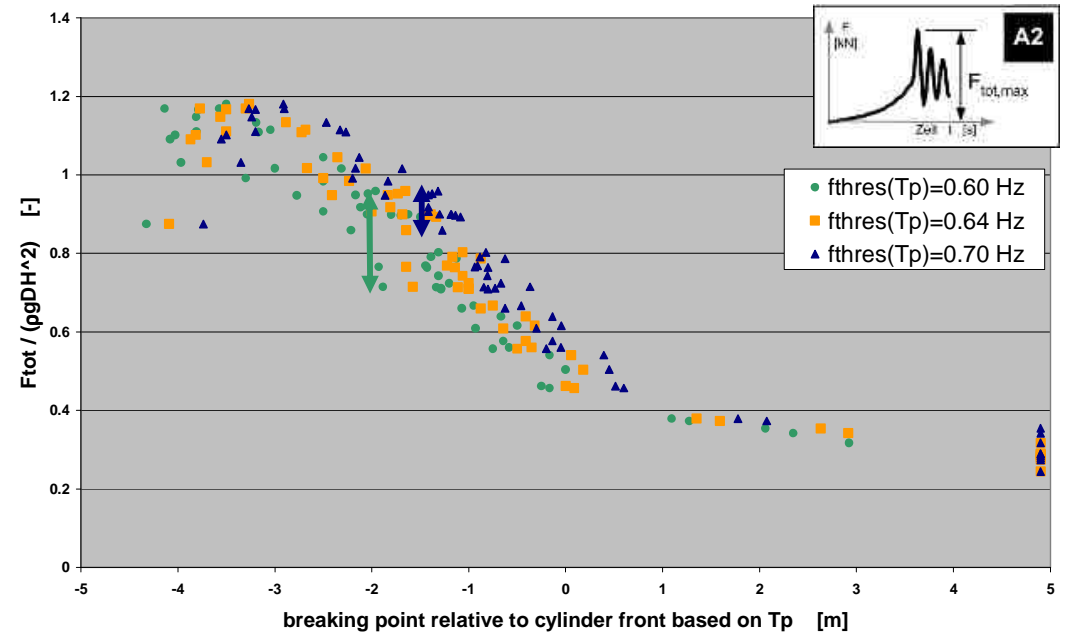

Figure 11. Non-dimensional measured total wave force against relative breaking point. Here PTM based on $T_{P}$ has been used.

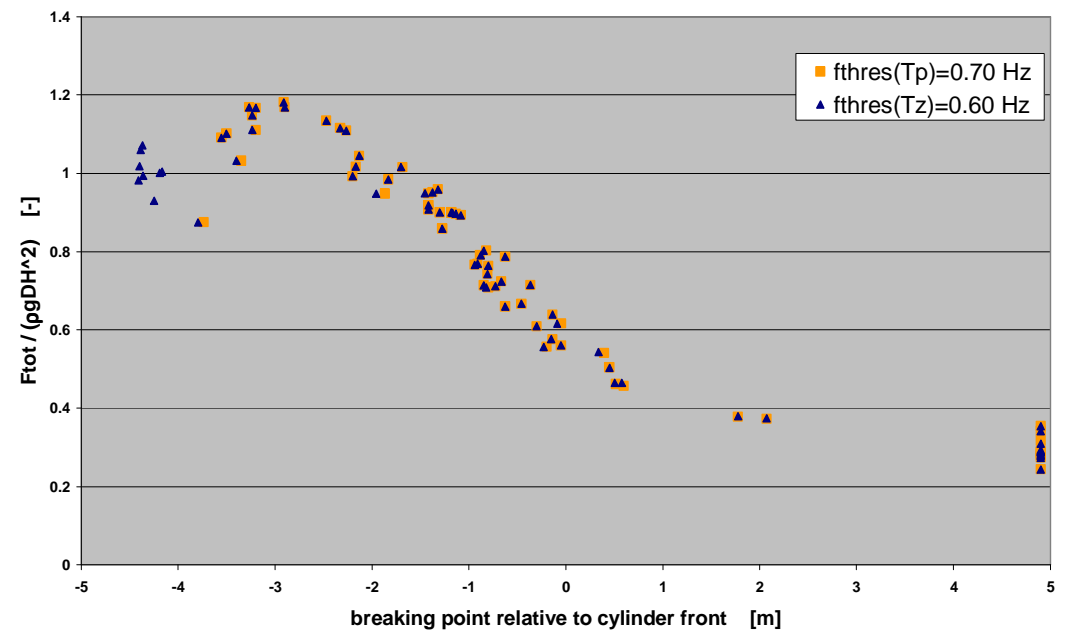

Figure 12. Comparison of the PTM results for threshold frequencies $f_{\text {thres }}\left(T_{p}\right)=0.7 \mathrm{~Hz}$ and $f_{\text {thres }}\left(T_{z}\right)=0.6 \mathrm{~Hz}$. 
The load case separation of the non-dimensional measured total wave force is performed in Figure 10 by using the PTM based on $T_{z}$. The breaking point is estimated for the previously determined $f_{\text {thres }}=0.60 \mathrm{~Hz}$ (see eq. 17) and additionally for $f_{\text {thres }}=0.50 / 0.55 \mathrm{~Hz}$. Depending on the threshold frequency, the separation results are much more detailed than the results of the visual analysis by Wienke \& Oumeraci (2005). For instance at $x=-2 \mathrm{~m}$ there is a high variation of the normalized force, if a threshold frequency of $f_{\text {thres }}=0.50 \mathrm{~Hz}$ is used. In contrast, the data follow almost a straight line for $f_{\text {thres }}=0.60 \mathrm{~Hz}$. This result confirms that

$$
f_{\text {thres }}\left(T_{z}\right)=0.60[\mathrm{~Hz}]
$$

is the optimal threshold frequency for the investigated conditions.

In Figure 11 the analysis of the wave force is based on the PTM with $T_{P}$. But now the use of the determined threshold $f_{\text {thres }}\left(T_{P}\right)=0.60 \mathrm{~Hz}$ (eq. 18) does not represent the best result. The best separation result is estimated with a threshold frequency just below the upper bound. According to Figure 11 the threshold frequency should be:

$$
f_{\text {thres }}\left(T_{P}\right)=0.70[\mathrm{~Hz}]
$$

Finally, the results of PTM by using eq. 19 and eq. 20 are shown in Figure 12 for comparison. There is hardly any difference detectable in the result. Only for the waves that hit the cylinder as broken waves, a clear difference is visible.

\section{SUMMARY AND CONCLUSIONS}

Laboratory experiments have been performed with breaking waves by using transient wave packets. Based on video records, the breaking points have been estimated visually. Then a breaking criterion based on the Phase-Time-Method (PTM) has been checked by the measured water surface elevation for different breaking wave conditions.

The threshold frequency for the PTM has been checked by using the zero down-crossing period $T_{z}$ (eq. 7) and the peak period $T_{P}$ (eq. 8). Due to the distinction of non-breaking and breaking waves the upper and lower bound of the threshold frequency have been identified.

The comparison with the visual analysis and with measured wave forces on a vertical cylinder confirms the same threshold frequency when using the zero down-crossing period $T_{z}$. The results differ for the peak period $T_{P}$. The application to the load case separation leads to the conclusion that the threshold frequency should be slightly lower than the upper bound:

$$
f_{\text {thres }}\left(T_{z}\right)=0.60[\mathrm{~Hz}]
$$

There is no clear recommendation if one should use the zero down-crossing period $T_{z}$ (eq. 7) or the peak period $T_{P}$ (eq. 8). Both results show excellent and robust results for transient wave packets.

The present analysis strengthens the overall confidence in the PTM and provides a more appropriate breaking criterion. Moreover, it also revealed some open issues for further research:

- Application of the threshold frequency for different breaker types,

- Maximum allowed distance between successive wave gauges, and

- Minimal sampling rate to achieve optimal results.

\section{ACKNOWLEDGMENTS}

The DFG supporting the basic research project "Belastung von zylindrischen Strukturen durch brechende und teilbrechende Wellen" (OU 1/4-2) is gratefully acknowledged by the first and third author. Many thanks are due to Jakob Stocker and Reinhold Schmidt-Koppenhagen for their support in the analysis of the experiments. 


\section{REFERENCES}

Bonmarin, P. 1989. Geometric properties of deep-water breaking waves. Journal of Fluid Mechanics, Vol. 209, No. 12, pp. 405-433.

Duncan, J.H.; Wallendorf, L.A.; Johnson, B. 1987. An experimental investigation of the kinematics of breaking waves. IAHR Seminar wave analysis and generation in laboratory basins; pp. 411-422.

Huang, N.E.; Long, S.R.; Tung, C.C.; Donelan, M.A.; Yuan, Y.; Lai, R.J. 1992. The local properties of ocean waves by the phase-time method. Geophysical Research Letters, Vol. 19, No. 7, pp. 685688.

Irschik, K., and H. Oumeraci. 2006. Effect of breaker types on breaking wave loads on a slender vertical and inclined pile. Proceedings of $30^{\text {th }}$ International Conference on Coastal Engineering, ICCE 2006, ASCE, 4520-4531.

Kamphuis, J.W. 1991. Incipient wave breaking, Coastal Engineering, Vol. 15, pp. 185-203.

Lader, P.F.; Grytoyr, G.; Myrhaug, D.; Peterrsen, B. 1998. Breaking wave geometry with emphasis on steepness and curvature. Ocean Wave Kinematics, Dynamics ans Loads on Structures; p. 281-288.

Wienke, J., and H. Oumeraci. 2005. Breaking wave impact force on a vertical and inclined slender pile - theoretical and large-scale model investigations. Coastal Engineering, 52 (5), 435-462.

Zimmermann, C.-A., and R. Seymour. 2002. Detection of Breaking in a Deep Water Wave Record. Journal of Waterway, Port, Coastal, and Ocean Engineering, ASCE, 128, 2, 72-78. 